\title{
A toxic error
}

Heavy metal pollution limits shouldn't just keep humans safe, but other animals too, say Coline Monchanin and Mathieu Lihoreau

ACROSS the world, insects are in decline. Intensive pesticide use, new diseases, habitat destruction and climate change are all contributing. Sadly, we are increasingly discovering that there is another impact of our everyday activities that is just as important: heavy metal pollution.

These substances are all around us. They are naturally present in Earth's crust and are released at low levels through weathering of rock and volcanic activity. But this gets a significant boost from human activity. We release these metals in various ways, ranging from dust that comes from vehicle brakes to the burning of fossil fuels for power and transport. All of this raises concentrations above natural levels. And once metallic dusts are out there, they stay for millennia.

While some of the compounds of these metals are essential for living organisms, most of them are highly toxic even at low concentrations. There are international guidelines designed to protect us from such pollution, but it turns out they aren't strong enough to do the same for insects.

In our recent work, we surveyed the scientific literature from the past 45 years that looked at the most monitored metals: arsenic, cadmium, mercury and lead. From this, we were able to identify the concentrations of these that are harmful to terrestrial invertebrates, the majority of which are insects. We then compared them with thresholds for the metals recommended by international regulatory bodies, for food, water and soil.

Though amounts of these in the environment should be below "human-safe" limits, in almost half of the studies the levels in natural conditions exceeded these figures. Not so surprisingly, at these concentrations the metals almost always killed invertebrates. More alarmingly, 90 per cent of the studies investigating metal levels within "humansafe" limits reported harmful effects on insects.

What's more, while it is clear that contamination harms these animals, we only have a partial picture as studies focus on sentinel species such as moths and bees, so-called because we use them to give an idea of possible harm to people, and on organisms favoured in many lab studies such as Drosophila. While we expect these to reflect a broad pattern, they only represent a tiny fraction of insect biodiversity, which is by far the most abundant of the terrestrial animals on the planet. A deeper understanding is critical to find the best possible solutions to mitigate these effects.

We need to start now, so we are calling for urgent action. While many local initiatives have worked to reduce emissions of some specific metal pollutants and emission sources over the past 40 years, environmental metallic pollution globally is still high. Take lead, for example, which started to be banned from petrol in the 1980s, but remains high in the environment because of the processing of ore and metals, and use of leaded aviation gasoline. 
We could effectively reduce metal emissions now by dramatically cutting the use of fossil fuels, gasoline vehicles and metal-based pesticides, which are all sensible ways to protect our environments.

Politicians and scientists also have to rethink guidelines about what a safe level of metal pollution is, to take account of non-human species. This kind of shift in thinking was achieved a few years ago for broad scale use of harmful pesticides in intensive agriculture in many parts of the world.

Metal pollution is still an underappreciated threat. Its potential contribution to the huge insect biodiversity decline, called "insectageddon" by some, hasn't been recognised in recent conservation plans, such as the proposal for a more sustainable European Union Common Agricultural Policy. It is time for that to change. 